Revista peruana de biología 26(1): 119 - 130 (2019) doi: http://dx.doi.org/10.15381/rpb.v26i1.15915 ISSN-L 1561-0837; elSSN: 1727-9933

Universidad Nacional Mayor de San Marcos

\section{Caracterización molecular de bacterias con potencial probiótico aisladas de heces de neonatos humanos}

\section{TRABAJOS ORIGINALES}

$\begin{array}{ll}\text { Presentado: } & 05 / 10 / 2018 \\ \text { Aceptado: } & 04 / 01 / 2019 \\ \text { Publicado online: } & 30 / 03 / 2019\end{array}$

\section{Correspondencia:}

1 Laboratorio de Ecología Microbiana y Biotecnología, Departamento de Biología, Facultad de Ciencias, Universidad Nacional Agraria La Molina, Lima-Perú.

2 Departamento Académico de Nutrición, Facultad de Zootecnia, Universidad Nacional Agraria La Molina, Lima-Perú.

* Autor de correspondencia:

Email EP: epaitananticona@gmail.com Email RS: rsantoa@gmail.com

Email AS: asotelo@lamolina.edu.pe Email DZ: dzuniga@lamolina.edu.pe Email CV: cvilchezp@lamolina.edu.pe

Citación:

Paitán E., R. Santos, A. Sotelo, D. Zúñiga, C. Vílchez. 2019. Caracterización molecular de bacterias con potencial probiótico aisladas de heces de neonatos humanos. Revista peruana de biología 26(1): 119 - 130 (Febrero 2019). doi: http://dx.doi. org/10.15381/rpb.v26i1.15915

Palabras clave: Recién nacidos; PCR-BOX; Enterococcus; Lactobacillus; Fermentar.

Keywords: Newborn; BOX-PCR; Enterococcus; Lactobacillus; ferment.

\section{Molecular characterization of bacteria with probiotic po- tential isolated from stool of human neonates}

\section{Elizabeth Paitán ${ }^{* 1,2}$, Ricardo Santos ${ }^{1}$, Alejandrina Sotelo ${ }^{2}$, Doris Zúñiga ${ }^{1}$, Carlos Vílchez ${ }^{2}$}

1 Facultad de Ciencias, Universidad Nacional Agraria La Molina, Lima-Perú. 2 Facultad de Zootecnia, Universidad Nacional Agraria La Molina, Lima-Perú.

\begin{abstract}
Resumen
El objetivo de este estudio es caracterizar molecularmente bacterias con potencial probiótico aisladas de heces de neonatos humanos. Se evaluó 60 muestras de heces de neonatos (0-3 días) se enriquecieron en caldo Man Rogosa y Sharp (MRS) a $37^{\circ} \mathrm{C} / 24 \mathrm{~h}$. Se seleccionó y se sometió a pruebas in vitro con sales biliares, resistencia a $\mathrm{pH}$ bajo y actividad antimicrobiana frente a Escherichia coli ATCC25922, E. coli ATCC35218, Salmonella enterica y Listeria inocua mediante el ensayo difusión en agar. La identificación molecular se realizó con amplificaciones PCR-BOX y el secuenciamiento del gen 16S rRNA. Se aislaron un total de 48 cepas y todas presentaron resistencia a $\mathrm{pH} 3$ y $0.3 \%$ sales biliares; 3 cepas mostraron actividad antimicrobiana frente a $E$. coli ATCC25922, 1 cepa frente a $E$. coli ATCC35218, 5 cepas frente a $L$. inocua y todas frente a Salmonella entérica. De las 48 cepas se obtuvieron dos perfiles BOX-PCR pertenecientes a los géneros de Lactobacillus y Enterococcus. Nueve cepas $\left(\mathrm{C}_{2}, \mathrm{C} 6 \mathrm{C}_{1}, \mathrm{C} 7, \mathrm{C} 11_{2}, \mathrm{C} 16_{2}, \mathrm{C} 19_{2}, \mathrm{C} 20, \mathrm{C} 35\right.$, y C42) presentaron un $100 \%$ de similaridad a Lactobacillus plantarum ATCC 14917 ${ }^{\top}$ [ACGZ01000098] y dos cepas (C15 y C40) un $99.93 \%$ y $99.80 \%$ de similaridad, respectivamente a Enterococcus faecium CGMCC 1.2136 ${ }^{\top}$ [AJKH01000109]; estas cepas mostraron actividad en leche con diferencias significativas ( $\mathrm{p}$ valor $<0.05$ ) en la cinética de $\mathrm{pH} 3$. En conclusión
\end{abstract} se encontró bacterias con potencial probiótico.

\section{Abstract}

The aim of this study is to molecularly characterize bacteria with probiotic potential isolated from feces of human neonates. Sixty stool samples from neonates (0-3 days) were evaluated and enriched in Man Rogosa and Sharp (MRS) broth at 37 ${ }^{\circ} \mathrm{C} / 24 \mathrm{~h}$. It was selected and subjected to in vitro tests with bile salts, resistance to low pH and antimicrobial activity against Escherichia coli ATCC25922, E. coli ATCC35218, Salmonella enterica and Listeria inocua by agar diffusion assay. The molecular identification was made with PCR-BOX amplifications and the sequencing of the $16 \mathrm{~S}$ rRNA gene. A total of 48 strains were isolated and all showed resistance to $\mathrm{pH} 3$ and $0.3 \%$ bile salts; 3 strains showed antimicrobial activity against $\mathrm{E}$. coli ATCC25922, 1 strain against E. coli ATCC35218, 5 strains against L. innocuous and all against $\mathrm{S}$. enterica. Of the 48 strains, two BOX-PCR profiles belonging to the genera of Lactobacillus and Enterococcus were obtained. Nine strains (C52, C61, C71, C112, C162, C192, C20, C35, and C42) presented 100\% similarity to L. plantarum ATCC 14917T [ACGZ01000098] and two strains (C15 and C40) 99.93\% and $99.80 \%$ similarity, respectively to Enterococcus faecium CGMCC 1.2136T [AJKH01000109]; these strains showed activity in milk with significant differences ( $p$ value $<0.05$ ) in the kinetics of $\mathrm{pH} 3$. In conclusion, bacteria with probiotic potential were found. 


\section{Introducción}

Enfermedades como la obesidad, diabetes tipo $1 \mathrm{y}$ 2 , colitis, hígado graso no alcohólico, y ciertos tipos de cáncer están asociadas con la alteración de la microbiota intestinal aberrante (Ley et al. 2006, Icaza 2013) y se ha demostrado que la modificación de esta puede prevenir $y$ tratar estas enfermedades (Tai et al. 2017).

La microbiota intestinal del ser humano está conformada por una carga microbiana de $10^{11}$ y $10^{12}$ bacterias por gramo de heces, dentro de los cuales se tienen aproximadamente 500 especies, encontrándose los lactobacilos en minoría (Visozo et al. 2006). La microbiota intestinal es fundamental para el correcto crecimiento corporal, la nutrición y el desarrollo de la inmunidad donde las alteraciones de esta podrían explicar en parte ciertas epidemias de la humanidad como el asma y la obesidad, así como la disbiosis que se ha asociado a diversos casos como el hígado graso no alcohólico, la enfermedad celíaca y el síndrome de intestino irritable (Icasaza 2013).

Los probióticos se definen como microorganismos viables o vivos que al ser consumidos en cantidades apropiadas confieren efectos benéficos para la salud del hospedero, siendo los más utilizados los géneros Lactobacillus y Bifidobacterium (FAO/OMS 2001, Delgado 2005). Asimismo, está probado el efecto beneficioso de los probióticos en alergias, infecciones urinarias, diarreas, e intolerancia a la lactosa, entre otros. Sin embargo, para que el uso de los probióticos sea exitoso, tienen que ser resistentes a las condiciones del tracto gastrointestinal, tales como las sales biliares y la acidez gástrica. Estos son criterios que debe cumplir cualquier microorganismo que se considere probiótico (FAO/OMS 2001, Delgado 2005, Cagigas et al. 2002, Mejía et al. 2007).

Es importante mencionar que la microbiota nativa está constituida por el conjunto de microorganismos que colonizan establemente la superficie de las mucosas, como las de la vagina de la mujer (Guarne 2007). Esta microbiota favorece la colonización del aparato digestivo del neonato durante el parto vaginal, como consecuencia del contacto del niño con la microbiota vaginal y perineal de la madre. Esta colonización se ve favorecida por el pH gástrico relativamente elevado y la secreción atenuada de bilis, típicas de los recién nacidos. La evolución posterior de la microbiota va a estar muy ligada al régimen alimenticio del niño, por lo cual la leche materna humana es otro factor importante en el desarrollo de la microbiota intestinal del neonato, ya que representa una fuente continua de microorganismos (Rodríguez et al. 2008, Olivares et al. 2008). Se ha observado que la microbiota de los lactantes alimentados con leche materna está constituida principalmente por bifidobacterias y lactobacilos, mientras que en la microbiota de los neonatos alimentados con fórmulas lácteas predominan las bacterias coliformes. (Ibañez 2017).

Esta microbiota nativa tiene gran importancia porque son bacterias que sirven de semilla para el desarrollo de la microbiota y son capaces de modular la colonización bacteriana neonatal con efecto protector sobre las enfermedades gastrointestinales infecciosas (La Rosa et. al. 2014). Hoy se tiene pruebas que las primeras colonias nativas funcionan como entrenadoras del sistema inmunitario y muchas de ellas son bacterias con potencial probiótico y por lo tanto son especies importantes por los beneficios en la salud del aparato digestivo humano y por la producción de compuestos antimicrobianos. Además, estas bacterias tienen la capacidad de fermentar diferentes carbohidratos y tienen alta tolerancia a $\mathrm{pH}$ bajo (Rodríguez 2009). Por ello las bacterias nativas de origen humano sirven de semilla o inoculo para cultivos benéficos a la salud por lo que es necesario caracterizarlos.

Los objetivos del presente estudio fueron: (1) caracterizar bacterias aisladas de heces de neonatos humanos, (2) evaluar su resistencia a pH 3, (3) sales biliares, así como su (4) actividad antimicrobiana frente a bacterias patógenas y que posean actividad en leche.

\section{Material y métodos}

Colección de las muestras.- Se colectaron 60 muestras aleatoriamente de heces de neonatos (de 0 a 3 días) nacidos en el Instituto Nacional Materno Perinatal (INMP) de Lima, desde noviembre del 2016 a febrero del 2017. Ésta colección fue en forma semanal (2 a 3 muestras) durante 4 meses, siguiendo el protocolo aprobado por la Comisión de Ética del INMP y con el material debidamente esterilizado, realizada la colección de la muestra se llevaron al laboratorio inmediatamente para su análisis.

Aspectos éticos.- El Comité de ética de Investigación del Instituto Nacional Materno Perinatal de Lima aprobó el proyecto (código 078-16).

Aislamiento de bacterias ácido lácticas.- Para el aislamiento de LAB se disolvió 2 gramos de cada muestra en $18 \mathrm{~mL}$ de caldo Man Rogose Sharpe (MRS) (Merck, USA) y se incubó a $37^{\circ} \mathrm{C}$ por 24 horas. Transcurrido este tiempo se realizaron diluciones seriadas hasta $10^{-6}$ usando para ello solución salina $(0.9 \% \mathrm{NaCl})$ y se vertieron en placas de agar MRS (Moreno et al. 2011) ajustado a pH 5.4 como medio selectivo para bacterias ácido lácticas (Rodríguez 2009), se incubo a $37{ }^{\circ} \mathrm{C}$ por 48 horas en microaerobiosis para el crecimiento bacteriano. Las colonias a aislar se sometieron a tinción Gram, detección de esporas y prueba de catalasa (Mejía et al. 2007). Con fines a diferenciar mejor morfológicamente las colonias se hizo una prueba adicional en MRS modificado (Lee \& Lee 2008) con bromofenol.

Las cepas aisladas se conservaron a $-80{ }^{\circ} \mathrm{C}$ (Cueto \& Aragón 2012) en caldo MRS con 40\% de glicerol como crio protector. Las bacterias se incubaron en caldo MRS a $37^{\circ} \mathrm{C}$ por 18 horas, previo a realizar los ensayos.

Resistencia a sales biliares.- Para este estudio se realizaron ensayos in vitro, donde la resistencia a sales biliares se hizo siguiendo la metodología utilizada por Lara y Burgos (2012), utilizando tubos con $3 \mathrm{~mL}$ de caldo MRS con adición de sales biliares 0,3\% de origen bovino (Sigma chemicol B8756), esterilizados en autoclave al 
que se inoculo con $10 \%$ de la cepa a evaluar y se incubo a $37{ }^{\circ} \mathrm{C}$ por 24 horas. Los controles fueron caldos a los cuales se sembró las cepas sin adición de sales biliares y para cuantificar la resistencia de las cepas se midió la densidad óptica (DO) a 600 nm (Ávila et al. 2010) en un espectrofotómetro UV (Génesis, 10S UV-VIS).

Tolerancia a ácido.- Para tolerancia a ácido se realizó en tubos con $3 \mathrm{~mL}$ de caldo MRS ajustada a pH 3 con adición de ácido clorhídrico 6M (Moreno et al. (2011), se inoculo $10 \%$ de la cepa a evaluar y se incubo a $37^{\circ} \mathrm{C}$ por 24 horas. Como referencia se usó caldo MRS sin adición de ácido clorhídrico, inoculado con la cepa a evaluar y se trató bajo los mismos parámetros de inoculación, temperatura y tiempo de incubación que se hicieron a pH 3.

Los resultados se expresaron como densidad óptica (DO) leída a 600 nm (Ávila et al. 2010) en un espectrofotómetro UV (Génesis, 10S UV-VIS). La tasa de sobrevivencia se calculó como el porcentaje de absorbancia (Sánchez et al. 2015) mediante la ecuación:

$$
\%=\frac{\text { Absorbancia } N_{1} \times 100}{\text { Absorbancia } N_{0}}
$$

$\mathrm{N}_{1}$ : Lectura de DO después del tratamiento

\section{$\mathrm{N}_{0}$ : Lectura de DO sin el tratamiento}

Las cepas con mayor tolerancia a sales biliares y resistencia a ácido durante las 24 horas se seleccionaron y sometieron a las mismas condiciones de $\mathrm{pH}$ y sales biliares durante 2 horas (Cueto et al. 2010) de incubación a $37^{\circ} \mathrm{C}$, seguidamente se realizaron las diluciones seriadas y siembra en placas con agar MRS dejando en incubación a $37^{\circ} \mathrm{C}$ por 24 horas para hacer el conteo de las colonias.

Actividad antimicrobiana.- La actividad antimicrobiana fue evaluada a las 48 cepas aisladas, y se determinó con la técnica de difusión en agar (Vallejo et al. 2009). Las bacterias indicadoras utilizadas fueron cepas de la Colección del Laboratorio de Microbiología Ecología y Biotecnología (Facultad de Ciencia, Biología - UNALM); estas bacterias fueron Escherichia coli ATCC25922, E. coli ATCC35218, Salmonella enterica y Listeria inocua, y como cepas control se utilizaron Lactobacillus plantarum y Lactococcus lactis.

Para esta prueba se preparó una suspensión a $60 \%$ de transmitancia de cada una de las 4 cepas indicadoras y se sembraron $30 \mu \mathrm{L}$ en placas de agar triptona de soya (TSA, OXOID, United Kingdom), se dejaron secar durante 5 minutos y en la superficie se depositó $5 \mu \mathrm{L}$ de los sobrenadantes de los cultivos libres de células bacterianas, las cuales previamente fueron centrifugadas a $5000 \mathrm{~g}$ durante 5 minutos. Se incubaron a $37^{\circ} \mathrm{C}$ por 24 horas, al término del cual se hicieron las lecturas correspondientes. Los resultados se expresaron como positivos cuando el halo externo formado fue mayor a $1 \mathrm{~mm}$ (Sánchez et al. 2015).

Caracterización molecular.- Las 48 cepas aisladas se activaron en caldo MRS a $37{ }^{\circ} \mathrm{C}$ por 18 horas, y se procedió a la extracción de DNA genómico de cada cepa de acuerdo a las indicaciones del kit comercial (Thermo SCIENTIFIC, USA) y la amplificación PCR fue realizado de acuerdo a Versalovic et al. (1991), empleando el primer BOX A1R (5'- CTACGGCAAGGCGACGCTGACG-3'). Obtenidos los perfiles BOX-PCR de las cepas en ensayo se formaron agrupamientos de alta similitud. Las cepas representativas de cada agrupamiento fueron amplificadas mediante el gen 16S rRNA usando cebadores (primers) fD1 GACAACAGAGTTTGATCCTGGCTCAG-3') y de reversa rD1 (5'-CCCGGGATCCAAGCTTAAGGAGGTGATCCAGCC-3') descritos por Versalovic et al. (1991). El producto de amplificación fue purificado con el kit (DNA Purification Kit-Thermo Scientific) y posteriormente secuenciado por MacroGen Inc. (Seoul, Korea).

Las lecturas y análisis de las secuencias se realizaron a partir de los cromatogramas y las secuencias de nucleótidos obtenidas se compararon con las bases de datos del GenBank PDB (BLASTN 2.2.0). Para la limpieza e identificación de las secuencias se usó el programa BioEdit Sequence Aligment Editor.

Cinética de crecimiento de las cepas ácido lácticas caracterizadas en leche.- Esta prueba se realizó con dos cepas representativas 5(2) y 15 que mostraron resistencia a medio adverso como $\mathrm{pH}$ bajo, sales biliares y actividad antimicrobiana. Se inició activando las cepas en tubos con $5 \mathrm{~mL}$ de caldo MRS a $37^{\circ} \mathrm{C}$ durante 24 horas.

Para determinar la cinética de crecimiento se siguió la técnica de Rodríguez (2009) con ciertas modificaciones, donde las cepas activadas en caldo se sembraron (10\%) en leche reconstituida (10\%), descremada y debidamente tindalizada, se dejaron en incubación a 37 ${ }^{\circ} \mathrm{C}$ de donde se tomó las muestras a las $0.0,3.0,6.0$, 9.0, 12.0 y 24 horas de incubación, para medir el pH y hacer la siembra en placas, por lo que se extrajo $1 \mathrm{~mL}$ de las muestras para hacer las diluciones hasta la $10^{-8} \mathrm{y}$ se sembró en placas con agar MRS, estos fueron incubados a 37 ${ }^{\circ} \mathrm{C}$ por 24 horas, al término de este tiempo se determinó el crecimiento log UFC/mL.

Todos los ensayos fueron por duplicado y los valores obtenidos se utilizaron para determinar la curva de crecimiento en leche.

Análisis estadístico.- Los resultados se analizaron utilizando el paquete SAS (Statistical Análisis System) versión 9 y se consideró diferencia estadísticamente significativa cuando $\mathrm{P}<0.05$.

\section{Resultados}

Aislamiento de bacterias ácido lácticas de heces.De las 60 muestras de heces de neonatos humanos de 0 a 3 días de nacidos en el INMP de Lima, se aislaron 48 cepas bacterianas con borde regular redondeado, con una elevación pequeña, de color blanco opaco, cuyo color se modificó a azulado cuando se sembró en agar MRS con bromofenol, pero se pudo diferenciar mejor por las características morfológicas de borde y elevación (Fig. 1). Estas colonias mostraron ser Gram positivas, no esporuladas, anaerobias facultativas y catalasa negativa. 

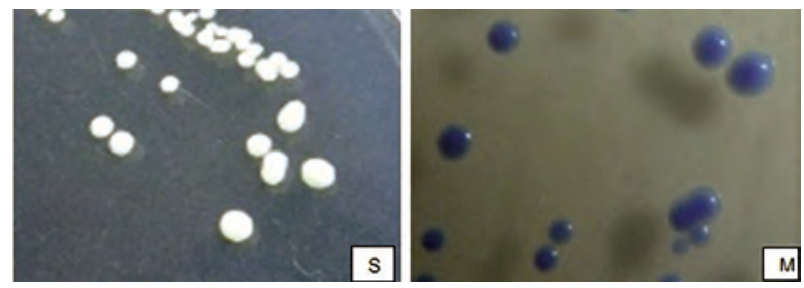

Figura 1. Colonias desarrolladas en agar MRS (S) y MRS (M).

Resistencia a sales biliares.- Es esencial que las bacterias tengan sistemas de protección a efectos de contrarrestar las condiciones del estómago y la bilis del intestino delgado, pues la digestión está en función al tipo de alimento además se debe considerar que el transito del alimento a través del intestino delgado es hasta de 12 horas (Clark \& Martin 1994). Por ello, con la finalidad de seleccionar cepas más resistentes se sometió a una incubación en caldo MRS con $0.3 \%$ de sales biliares a 37 ${ }^{\circ} \mathrm{C}$ durante 24 horas, en la que la totalidad de las cepas mostraron resistencia aunque algunas tuvieron mayor resistencia respecto al control (Tabla 1). En total se aislaron 15 cepas $(31.25 \%)$ que presentaron una resistencia igual o mayor a $30 \%$ a la concentración de sales biliares respecto al control.

Tolerancia a pH bajo.- La resistencia a pH bajo dio como resultado diversos porcentajes de tolerancia a estas condiciones, donde algunas cepas mostraron mejor resistencia que otras a pesar que todas las cepas resistieron dichas condiciones; sin embargo, solo se consideró como positivo a aquellas cepas que tuvieron $30 \%$ o más de tolerancia en medio acido respecto al de la referencia obteniéndose una toleración del 60.4\% (29 cepas, Tabla 2).

Comparando los porcentajes de sobrevivencia de las 48 cepas sometidas a pH 3 y resistencia a $0.3 \%$ de sales biliares, se encontró diferencias significativas $(\mathrm{p}<0.05)$ donde las cepas aisladas presentaron mejor tolerancia al pH en comparación a las sales biliares.

Se seleccionaron 11 cepas que tuvieron mejor tolerancia (30\% mínimo) a $0.3 \%$ de sales biliares y a $\mathrm{pH} 3$ (50\% mínimo, con ácido clorhídrico), siendo estas sometidas a las mismas condiciones en caldo MRS ajustado a pH 3 y caldo con $0.3 \%$ de sales biliares a $37{ }^{\circ} \mathrm{C}$ durante dos horas de incubación, obteniéndose buen crecimiento de 3 cepas 5(2), 6(1) y 40 a pH 3 que fueron igual a la cepa de referencia L. plantarum (7 log UFC/mL) mientras que las demás cepas tuvieron un crecimiento de 6 $\log \mathrm{UFC} / \mathrm{mL}$.

La tolerancia a $0.3 \%$ de sales biliares de las cepas 5(2), 6(1), 19, 20 y 40 mostraron el mismo crecimiento que la cepa de referencia (L. plantarum, $5 \log \mathrm{UFC} / \mathrm{mL}$ ) y en las cepas restantes el crecimiento fue $1 \log \mathrm{UFC} / \mathrm{mL}$ menos que la cepa de referencia (Tabla 3).

Un criterio obligatorio en la selección de cepas probióticas para la producción de alimentos probióticos, como los productos lácteos fermentados y otros, es la capacidad de sobrevivir a condiciones de $\mathrm{pH}$ bajo y resistir a las sales biliares. En este estudio se encontró que la mayoría de las cepas evaluadas mostraron mejor resisten-
Tabla 1. Densidad óptica de sobrevivencia de cepas sometidas a $\mathrm{pH} 3$ y $0.3 \%$ de sales biliares a $37^{\circ} \mathrm{C}$ durante 24 horas.

\begin{tabular}{|c|c|c|c|}
\hline Codigo & Referencia & $\mathrm{pH} 3$ & S. biliares $0.3 \%$ \\
\hline $1(2)$ & 1.860 & 0.7820 & 0.320 \\
\hline 2 & 1.850 & 0.7950 & 0.041 \\
\hline $3(2)$ & 1.878 & 1.3000 & 0.650 \\
\hline $4(1)$ & 1.891 & 0.7100 & 0.660 \\
\hline $4(2)$ & 2.170 & 0.5000 & 0.600 \\
\hline $5(2)$ & 1.892 & 0.9520 & 0.650 \\
\hline $6(1)$ & 1.859 & 0.6800 & 0.500 \\
\hline $7(1)$ & 1.820 & 0.7700 & 0.600 \\
\hline $7(2)$ & 1.876 & 0.8700 & 0.600 \\
\hline 8 & 1.831 & 0.5280 & 0.060 \\
\hline $9(1)$ & 1.879 & 0.4100 & 0.030 \\
\hline 10 & 1.825 & 0.4350 & 0.030 \\
\hline $11(2)$ & 1.890 & 0.4040 & 0.250 \\
\hline 12 & 1.860 & 0.4300 & 0.300 \\
\hline 13 & 1.860 & 0.3000 & 0.030 \\
\hline 14 & 1.980 & 0.0300 & 0.040 \\
\hline 15 & 1.870 & 0.9500 & 0.360 \\
\hline $16(2)$ & 1.910 & 0.9320 & 0.630 \\
\hline 17 & 1.910 & 0.8500 & 0.540 \\
\hline 18 & 1.880 & 0.6350 & 0.640 \\
\hline 19(1) & 1.820 & 1.1200 & 0.600 \\
\hline $20(2)$ & 1.870 & 1.0560 & 0.700 \\
\hline $21(1)$ & 1.950 & 1.2200 & 0.160 \\
\hline $22(1)$ & 1.820 & 0.9190 & 0.450 \\
\hline $23(1)$ & 1.900 & 0.5470 & 0.520 \\
\hline 24 & 1.860 & 0.4150 & 0.030 \\
\hline $25(2)$ & 1.860 & 1.0300 & 0.950 \\
\hline 26 & 1.890 & 0.8540 & 0.450 \\
\hline 27 & 1.940 & 0.4500 & 0.050 \\
\hline 28 & 1.960 & 0.4500 & 0.700 \\
\hline 29 & 1.890 & 0.1210 & 0.600 \\
\hline $30(1)$ & 2.170 & 0.2220 & 0.700 \\
\hline $30(2)$ & 1.890 & 0.4752 & 0.380 \\
\hline $31(1)$ & 1.890 & 0.9500 & 0.750 \\
\hline $32(2)$ & 1.830 & 0.0600 & 0.600 \\
\hline $33(2)$ & 1.930 & 0.6400 & 0.400 \\
\hline 34 & 1.890 & 0.6200 & 0.100 \\
\hline 35 & 1.860 & 0.5410 & 0.400 \\
\hline 36 & 1.892 & 0.8240 & 0.240 \\
\hline 37 & 1.850 & 0.9100 & 0.400 \\
\hline 38 & 1.820 & 0.1240 & 0.380 \\
\hline 39 & 1.920 & 0.9800 & 0.060 \\
\hline $40(1)$ & 1.960 & 1.0300 & 0.450 \\
\hline $41(1)$ & 1.822 & 0.1090 & 0.100 \\
\hline 42 & 1.890 & 0.9900 & 0.100 \\
\hline 43 & 1.852 & 0.8200 & 0.100 \\
\hline $44(1)$ & 1.830 & 0.8350 & 0.240 \\
\hline $45(2)$ & 1.890 & 0.6590 & 0.210 \\
\hline
\end{tabular}


Tabla 2. Porcentaje de sobrevivencia de cepas después de las 24 $\mathrm{h}$ de incubación a sales biliares y pH bajo.

\begin{tabular}{|c|c|c|}
\hline Código & $\mathrm{pH} 3$ & $0.3 \%$ Sales biliares \\
\hline $1(2)$ & 42 & 17 \\
\hline $2(2)$ & 43 & 2 \\
\hline $3(2)$ & 69 & 35 \\
\hline $4(1)$ & 38 & 35 \\
\hline $4(2)$ & 23 & 28 \\
\hline $5(2)$ & 50 & 34 \\
\hline $6(1)$ & 37 & 27 \\
\hline $7(1)$ & 42 & 33 \\
\hline $7(2)$ & 46 & 32 \\
\hline 8 & 29 & 3 \\
\hline $9(1)$ & 22 & 2 \\
\hline 10 & 24 & 2 \\
\hline $11(2)$ & 21 & 13 \\
\hline 12 & 23 & 16 \\
\hline 13 & 16 & 2 \\
\hline 14 & 2 & 2 \\
\hline 15 & 51 & 19 \\
\hline $16(2)$ & 49 & 33 \\
\hline 17 & 45 & 28 \\
\hline 18 & 34 & 34 \\
\hline 19(1) & 62 & 33 \\
\hline $20(2)$ & 56 & 37 \\
\hline 21(1) & 62 & 8 \\
\hline $22(1)$ & 50 & 25 \\
\hline $23(1)$ & 29 & 27 \\
\hline 24 & 22 & 2 \\
\hline $25(2)$ & 55 & 51 \\
\hline 26 & 45 & 24 \\
\hline 27 & 23 & 3 \\
\hline 28 & 23 & 36 \\
\hline 29 & 6 & 32 \\
\hline $30(1)$ & 10 & 32 \\
\hline $30(2)$ & 25 & 20 \\
\hline $31(1)$ & 50 & 40 \\
\hline $32(2)$ & 3 & 33 \\
\hline $33(2)$ & 33 & 21 \\
\hline 34 & 33 & 5 \\
\hline 35 & 29 & 22 \\
\hline 36 & 44 & 13 \\
\hline 37 & 49 & 22 \\
\hline 38 & 7 & 21 \\
\hline 39 & 51 & 3 \\
\hline $40(1)$ & 53 & 23 \\
\hline $41(1)$ & 6 & 5 \\
\hline 42 & 52 & 5 \\
\hline 43 & 44 & 5 \\
\hline $44(1)$ & 46 & 13 \\
\hline $45(2)$ & 35 & 11 \\
\hline
\end{tabular}

$(p<0.05)$
Tabla 3. Efecto de la tolerancia a pH bajo y sales biliares sobre el crecimiento microbiano

\begin{tabular}{ccc}
\hline Código & $\begin{array}{c}\mathrm{pH} 3 \\
\log \text { UFC/mL }\end{array}$ & $\begin{array}{c}0.3 \% \text { sales biliares } \\
\log \text { UFC } / \mathrm{mL}\end{array}$ \\
\hline $5(2)$ & 7 & 5 \\
$6(1)$ & 7 & 5 \\
$7(1)$ & 7 & 4 \\
$11(2)$ & 6 & 4 \\
15 & 6 & 4 \\
16 & 6 & 4 \\
19 & 6 & 5 \\
20 & 6 & 5 \\
35 & 6 & 4 \\
40 & 7 & 5 \\
42 & 6 & 4 \\
L. plantarum & 7 & 5 \\
\hline
\end{tabular}

cia a pH 3 que tolerancia a sales biliares, lo que sugiere que podrían sobrevivir al $\mathrm{pH}$ bajo del estómago, por lo que, las cepas 5(2), 6(1), 7(1) y 40 cumplieron con este criterio debido a que mostraron buen número de células viables en esta prueba.

Actividad antimicrobiana .- En la prueba de actividad antimicrobiana contra microorganismos patógenos se observó la formación de halos mayores a $1 \mathrm{~mm}$. En la Tabla 4 se puede apreciar que todas las cepas mostraron inhibición contra $S$. entérica, mientras que sólo tres cepas 5(2), 6(1) y 7(1) inhibieron E. coli ATCC25922; una cepa 7(1) inhibió E. coli ATCC35218 y cinco cepas 5(2), 7(1), 7(2), 11 y 15 inhibieron L. inocua (Fig. 2).

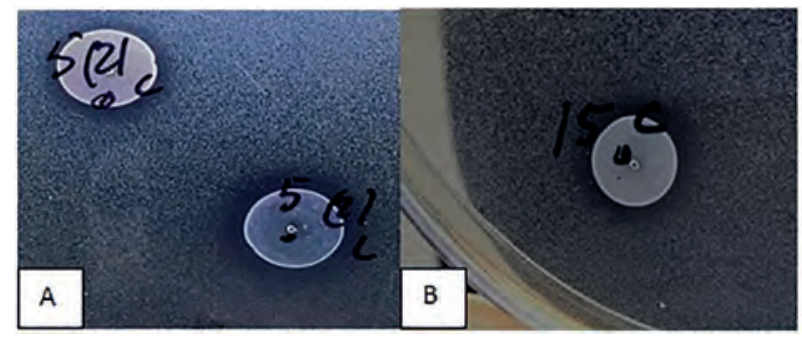

Figura 2. Ensayo de difusión en agar de las cepas aislados de neonatos contra Listeria inocua. (A). Cepa 5(2)c con caldo MRS y 5(2)L con leche. (B). Cepa 15 con caldo MRS.

La actividad antimicrobiana de las cepa 7(1) fue positiva contra todas las bacterias patógenas indicadoras E. coli ATCC25922, E. coli ATCC35218, L. inocua y $S$. entérica; mientras que la cepa 5(2) mostró actividad antimicrobiana contra tres bacterias indicadoras $E$. coli ATCC25922, L. inocua y $S$. enterica, pero no contra E. coli ATCC35218. Y la cepa 15 y 11 tuvo actividad antimicrobiana contra dos cepa L. inocua y S. enterica (Tabla 4).

Las cepas fueron activadas en leche previo a la prueba de actividad antimicrobiana, observandose que la cepa 5(2)L mostró un halo mas grande contra L. inocua en comparacion a dicha cepa activada en caldo MRS. Ade- 
Tabla 4. Actividad antimicrobiana de las cepas aisladas de neonatos contra a E. coli ATCC25922, E. coli ATCC35218, L. inocua y S. enterica.

\begin{tabular}{|c|c|c|c|c|}
\hline Código & $\begin{array}{c}\text { E. coli } \\
\text { ATCC25922 }\end{array}$ & $\begin{array}{c}\text { E. coli } \\
\text { ATCC35218 }\end{array}$ & L. inocua & S. entérica \\
\hline $1(2)$ & --- & --- & --- & +++ \\
\hline $2(2)$ & --- & --- & --- & +++ \\
\hline $3(2)$ & --- & --- & --- & +++ \\
\hline $4(1)$ & --- & --- & --- & +++ \\
\hline $4(2)$ & --- & --- & --- & +++ \\
\hline $5(2)$ & +++ & --- & +++ & +++ \\
\hline $6(1)$ & +++ & --- & --- & +++ \\
\hline $7(1)$ & +++ & +++ & +++ & +++ \\
\hline $7(2)$ & ++- & - & +++ & +++ \\
\hline 8 & --- & --- & --- & +++ \\
\hline $9(1)$ & --- & --- & --- & +++ \\
\hline 10 & --- & --- & --- & +++ \\
\hline $11(2)$ & --- & --- & +++ & +++ \\
\hline 12 & --- & --- & --- & +++ \\
\hline 13 & --- & --- & -++ & +++ \\
\hline 14 & --- & --- & --- & +++ \\
\hline 15 & --- & --- & +++ & +++ \\
\hline $16(2)$ & --- & --- & --- & +++ \\
\hline 17 & --- & --- & --- & +++ \\
\hline 18 & --- & --- & --- & +++ \\
\hline 19(1) & --- & --- & --- & +++ \\
\hline $20(2)$ & --- & --- & --- & +++ \\
\hline $21(1)$ & --- & --- & --- & +++ \\
\hline $22(1)$ & --- & --- & --- & +++ \\
\hline $23(1)$ & --- & --- & --- & +++ \\
\hline 24 & --- & --- & --- & +++ \\
\hline $25(2)$ & --- & --- & --- & +++ \\
\hline 26 & --- & --- & --- & +++ \\
\hline 27 & --- & --- & --- & +++ \\
\hline 28 & --- & --- & --- & +++ \\
\hline 29 & --- & --- & --- & +++ \\
\hline $30(1)$ & --- & --- & --- & +++ \\
\hline $30(2)$ & --- & --- & --- & +++ \\
\hline $31(1)$ & --- & --- & --- & +++ \\
\hline $32(2)$ & --- & --- & --- & +++ \\
\hline $33(2)$ & --- & --- & --- & +++ \\
\hline 34 & --- & --- & --- & +++ \\
\hline 35 & --- & --- & --- & +++ \\
\hline 36 & --- & --- & --- & +++ \\
\hline 37 & --- & --- & --- & +++ \\
\hline 38 & --- & --- & --- & +++ \\
\hline 39 & --- & --- & --- & +++ \\
\hline 40(1) & --- & --- & -++ & +++ \\
\hline $41(1)$ & --- & --- & --- & +++ \\
\hline 42 & --- & --- & --- & +++ \\
\hline 43 & --- & --- & --- & +++ \\
\hline $44(1)$ & --- & --- & --- & +++ \\
\hline $45(2)$ & --- & --- & --- & +++ \\
\hline
\end{tabular}

mas, la cepa 15 activada en caldo MRS mostró presencia de halo contra L. inocua (Fig. 2).

Las cepas control A (L. plantarum y L. lactis) utilizadas en esta prueba formaron halos similares a aquellos formados por las cepas en estudio (B) contra S. entérica (Fig. 3).

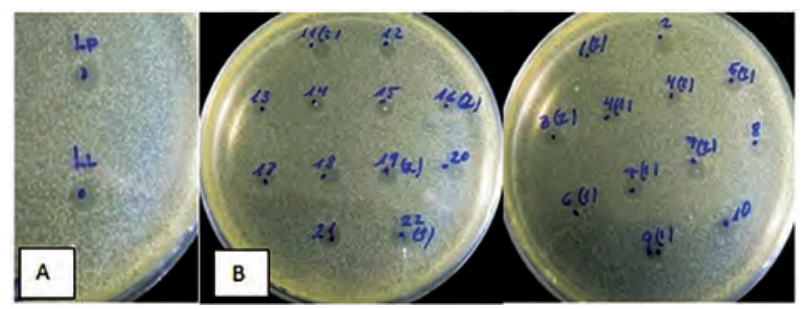

Figura 3. Ensayo de difusión en agar de las cepas aisladas de neonatos contra Salmonella enterica. (A). Cepas control: Lp= Lactobacillus plantarum y LI= Lactococcus lactis. (B). cepas aisladas de neonatos.

\section{Identificación molecular de las cepas aisladas de neonatos}

Amplificación PCR-BOX.- Para conocer la diversidad microbiana de las bacterias acido lácticas aisladas de neonatos humanos se extrajo el ADN y se amplificó las 48 cepas obteniéndose perfiles similares tal como se muestra en la Figura 4. Cada uno de los perfiles fue analizado de acuerdo a las bandas formadas obteniéndose dos grupos diferentes.

Secuenciación del gen $16 S$ rRNA.- Para ello, se seleccionaron 11 cepas que cumplían criterios de tolerancia a $\mathrm{pH} 3$, resistencia a $0.3 \%$ de sales biliares y actividad antimicrobiana. En la Figura 5 se pueden apreciar las bandas del gen $16 \mathrm{~S}$ de las once cepas.

Comparados y analizados las secuencias con la base de datos del GenBank se encontró nueve cepas [5(2), 6(1), 7(1), 11(2), 16(2), 19 (2), 20, 35 y 42] con 100\% de similaridad a Lactobacillus plantarum ATCC $14917^{\mathrm{T}}$ y dos cepas (15 y 40) con $99.93 \%$ y $99.80 \%$ de similaridad a Enterococcus faecium CGMCC $12136^{\mathrm{T}}$ respectivamente. En este estudio se aisló y caracterizo mayor número de lactobacilos que lactococos (Tabla 5).

En las 11 cepas clasificadas filogénicamente aisladas de heces de neonatos humanos nacidos en el INMP de Lima se halló mayor número de lactobacilos (9 cepas) que lactococos(2 cepas), donde la cepa 5(2) (Fig. 6) mostró buen potencial probiótico, mientras que, para los lactococos la cepa 15 mostró potencial probiótico.

Cinética de crecimiento de las cepas en leche.- La cinética de crecimiento en leche de la cepa 5(2), que tuvo un $100 \%$ de similaridad a L. plantarum subsp. plantarum ATCC 14917T, desarrolló una rápida multiplicación que se incrementó de 5 a 7 log UFC/mL en tres horas, y alcanzando $8 \log$ UFC/mL a las 9 horas, evidenciando así un buen crecimiento de esta bacteria antes de llegar a la fase estacionaria (Fig. 7).

La cinética de crecimiento de la cepa 15, que tuvo 99.93\% de similaridad a Enterococcus faecium CGMCC 

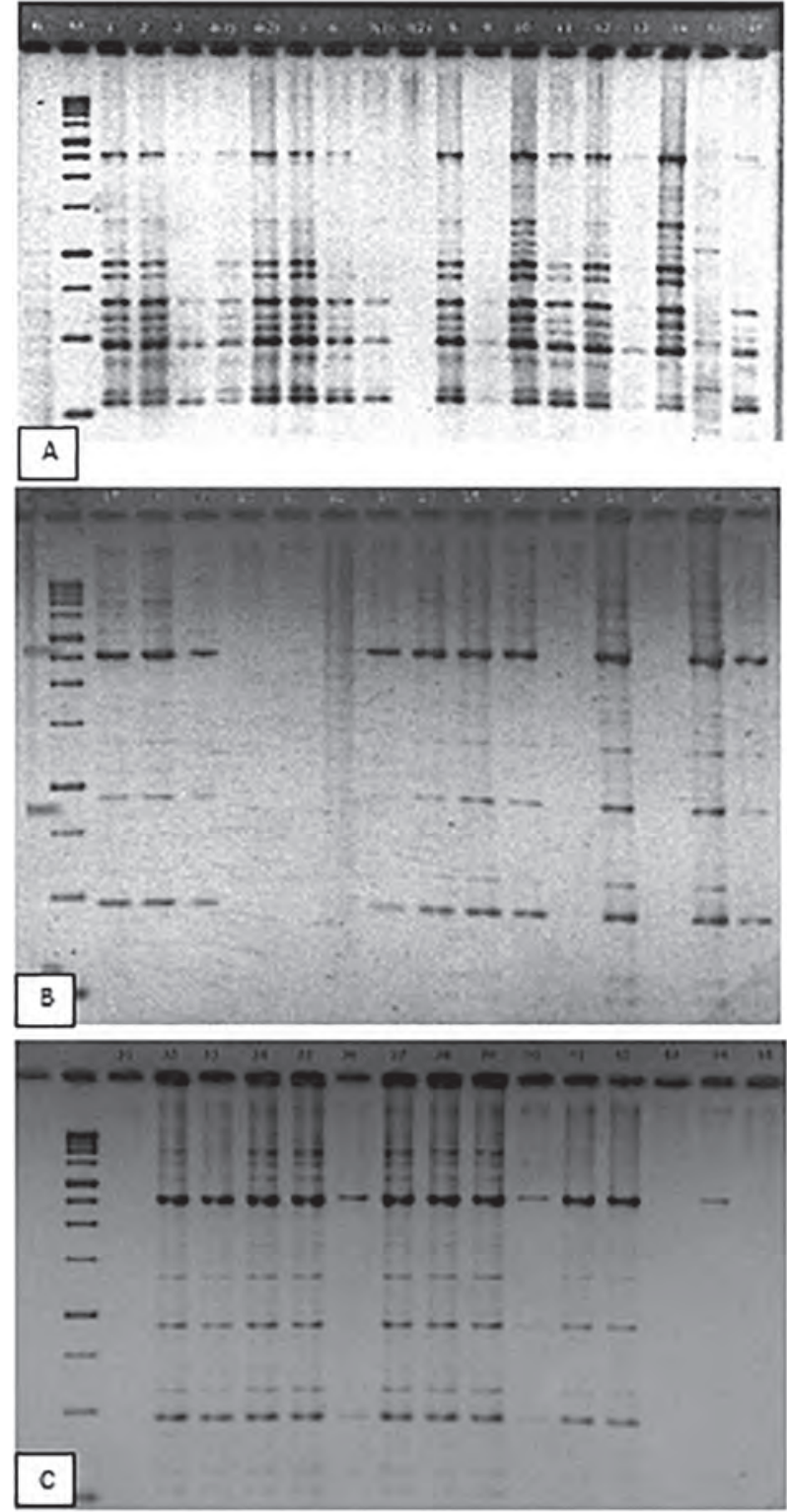

Figura 4. Perfiles PCR-BOX de las cepas aisladas de neonatos usando el primer BOXA1R para perfiles: (A) de 1 al 16, (B) del 17 al 31, y (C) del 32 al 45 .

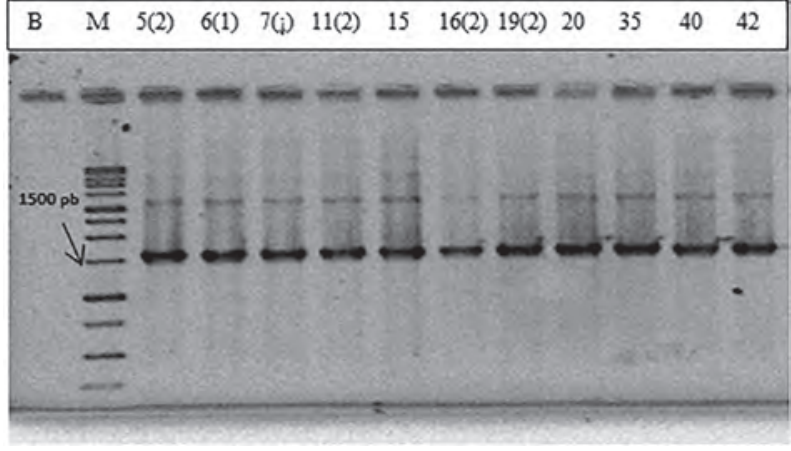

Figura 5. Electroforesis del gen 16S rRNA de once cepas seleccionadas. B: blanco, M: Marcador $1 \mathrm{~Kb}$.
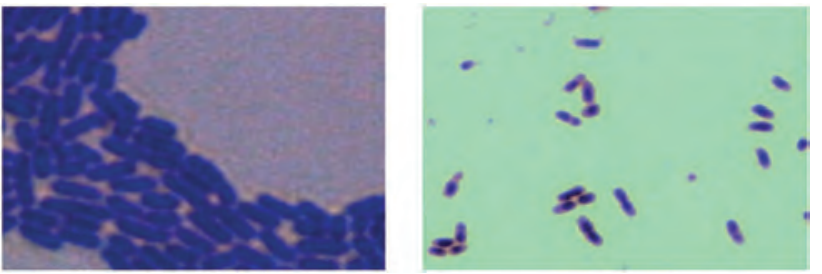

Figura 6. Observación al microscopio 5(2) de Lactobacillus plantarum subsp. plantarum ATCC $14917^{\top}$ (Izquierda) y 15 Enterococcus faecium CGMCC $1.2136^{\top}$ (Derecha).

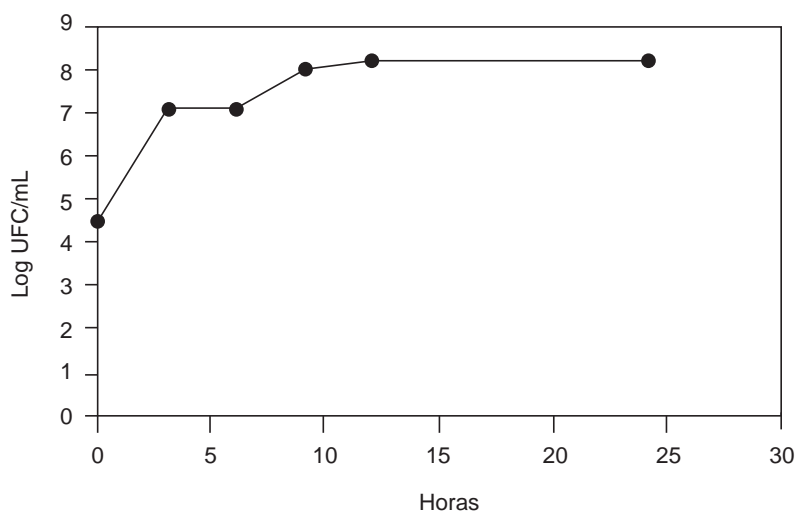

Figura 7. Cinética de crecimiento respecto al tiempo en leche de la cepa 5(2) con 100\% de similaridad a Lactobacillus plantarum subsp. plantarum ATCC $14917^{\top}$

Tabla 5. Caracterización molecular y clasificación filogenética de las cepas BAL aisladas de neonatos humanos.

\begin{tabular}{|c|c|c|c|c|}
\hline Código de cepa & Agrupamiento & Identificación molecular por secuenciamiento del gen ribosomal 16s & Número de Accesión & Similaridad (\%) \\
\hline $5(2)$ & A & Lactobacillus plantarum subsp. plantarum ATCC $14917^{\top}$ & ACGZ01000098 & 100 \\
\hline $6(1)$ & A & Lactobacillus plantarum subsp. plantarum ATCC $14917^{\top}$ & ACGZ01000098 & 100 \\
\hline $7(1)$ & A & Lactobacillus plantarum subsp. plantarum ATCC $14917^{\top}$ & ACGZ01000098 & 100 \\
\hline $11(2)$ & A & Lactobacillus plantarum subsp. plantarum ATCC $14917^{\top}$ & ACGZ01000098 & 100 \\
\hline 15 & B & Enterococcus faecium CGMCC $1.2136^{\top}$ & AJKH01000109 & 99.93 \\
\hline $16(2)$ & A & Lactobacillus plantarum subsp. plantarum ATCC $14917^{\top}$ & ACGZ01000098 & 100 \\
\hline 19(2) & A & Lactobacillus plantarum subsp. plantarum ATCC $14917^{\top}$ & ACGZ01000098 & 100 \\
\hline 20 & A & Lactobacillus plantarum subsp. plantarum ATCC $14917^{\top}$ & ACGZ01000098 & 100 \\
\hline 35 & A & Lactobacillus plantarum subsp. plantarum ATCC $14917^{\top}$ & ACGZ01000098 & 100 \\
\hline 40 & B & Enterococcus faecium CGMCC $1.2136^{\top}$ & AJKH01000109 & 99.80 \\
\hline 42 & A & Lactobacillus plantarum subsp. plantarum ATCC $14917^{\top}$ & ACGZ01000098 & 100 \\
\hline
\end{tabular}


1.2136T, mostró rápido crecimiento pasando de 5 a 7 $\log \mathrm{UFC} / \mathrm{mL}$ al cabo de tres horas, llegando hasta 8 Log $\mathrm{UFC} / \mathrm{mL}$ a las 24 horas. Lo que evidencia que el mayor crecimiento de estas bacterias se produce dentro de las 3 primeras horas de incubación (Fig. 8)

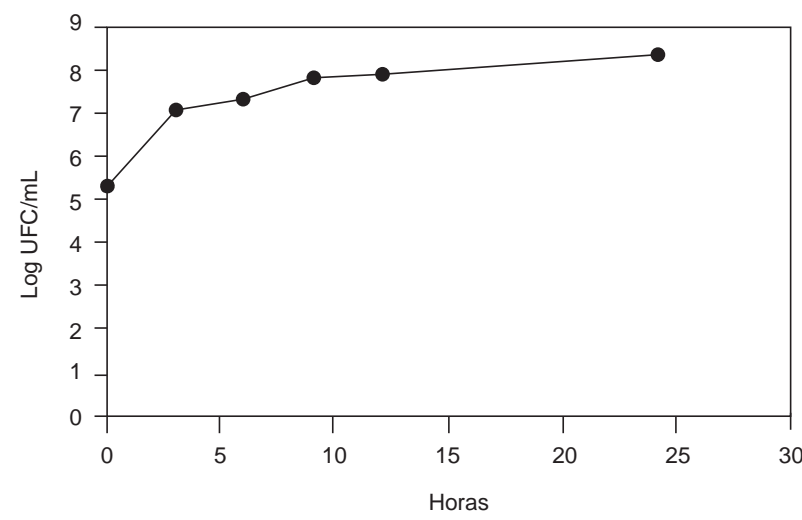

Figura 8. Cinética de crecimiento respecto al tiempo en leche de la cepa 15 con $99.93 \%$ de similaridad a Enterococcus faecium CGMCC $1.2136^{\top}$

En la Tabla 6 se muestra la variación de pH y el crecimiento de las cepas 5(2) y 15 durante las 24 horas de incubación. La cinética de disminución de pH es un factor importante para la selección de bacterias ácidos lácticas en la producción de productos lácteos fermentados, siendo las cepas 5(2) y 15 las que mostraron mejor actividad de fermentación en leche, determinándose diferencias significativas $(\mathrm{p}<0.05)$ en la cinética de $\mathrm{pH}$ a favor de la cepa 15 como mejor productor de ácido láctico.

Tabla 6. Variación del pH y crecimiento de las cepas en leche.

\begin{tabular}{cccccc}
\hline & \multicolumn{2}{c}{ Cepa 5(2) } & & \multicolumn{2}{c}{ Cepa 15 } \\
\cline { 2 - 3 } \cline { 5 - 6 } Horas & $\mathrm{pH}$ & Log UFC/mL & & $\mathrm{pH}$ & Log UFC/mL \\
\hline 0 & 5.48 & 4.50 & & 5.50 & 5.30 \\
3 & 5.40 & 7.10 & & 5.35 & 7.10 \\
6 & 4.83 & 7.20 & & 4.91 & 7.30 \\
9 & 4.49 & 8.00 & & 4.66 & 7.80 \\
12 & 4.14 & 8.10 & & 4.22 & 7.85 \\
24 & 3.68 & 8.20 & & 3.65 & 8.30 \\
\hline$(p<0.05)$ & & & &
\end{tabular}

\section{Discusión}

Aislamiento de bacterias ácido lácticas de heces.A través de las diferentes investigaciones se han podido identificar diversas ventajas del microorganismo probióticos, lo que ha incrementado sobretodo el consumo de productos lácteos fermentados, siendo importante identificar y caracterizar estas bacterias a través de aspectos como características morfológicas, fisiológicas y técnicas moleculares.

En el presente estudio las características morfológicas de las colonias en agar MRS coinciden con colonias típicas formadas por BAL (Yadav et al. 2016). Todas las bacterias aisladas fueron Gram-positivas, catalasa-nega- tiva, no esporulados, anaerobios facultativos y durante la fermentación no produjeron gas; estos correspondían a bacilos y algunos lactococos, encontrándose estas últimas con células en pares. Shin et al. (2008) reportan iguales características de bacterias aisladas del tracto gastrointestinal de pollos. Los lactococos son comensales habitantes del tracto gastrointestinal del ser humano $\mathrm{y}$ animales, que frecuentemente se encuentran en alimentos fermentados Ren et al. (2016).

Resistencia a sales biliares.- La tolerancia a sales biliares es considerada una prueba esencial para las bacterias ácido lácticas a efectos que puedan sobrevivir al paso del intestino; en nuestro estudio el uso de $0.3 \%$ de sales biliares redujo el crecimiento en $1 \mathrm{Log} / \mathrm{mL}$ en 6 de las cepas caracterizadas y en $5 \mathrm{Log} / \mathrm{mL}$ en 11 cepas, cuyo comportamiento fue similar al control (Lactobacillus plantarum) reforzando así lo reportado por Park et al. (2002). Las bacterias ácido lácticas probióticas varían su nivel de tolerancia biliar considerablemente, sin embargo se desconoce el mínimo nivel de tolerancia biliar que estos poseen (Klaenhammer 1982).

Las cepas que mostraron mayor resistencia a las sales biliares podría deberse a que hidrolizan mejor las sales biliares a través de la enzima hidrolasa biliar atenuando de esta manera el efecto detergente de la sal biliar (Erkkila \& Petaja, 2000). Las bacterias ácido lácticas que tuvieron resistencia a concentraciones de sales biliares $0.3 \%$ permite seleccionar cepas resistentes para el uso en productos fermentados por probióticos (Erkkila \& Petaja 2000).

Cueto y Aragón (2012) obtuvieron mayor porcentaje de crecimiento de bacterias ácido lácticas a pH bajo (3.0) comparado con aquellas sometidas a concentraciones de $0.3 \%$ de sales biliares, coincidiendo con lo hallado en el presente estudio.

Tolerancia a pH bajo.- La tolerancia a pH bajo es otra característica importante de los probióticos (Brink et al. 2006). El recubrimiento de sustancias poliméricas extracelulares (EPS) puede ser un protector de las bacterias que les permite soportar el ácido del estómago (Boke et al. 2010); de acuerdo a nuestros resultados algunas cepas estarían mejor recubiertas de EPS que otras, por ello, la DO mostraron valores diferentes de tolerancia a pH bajo. Estos resultados coinciden con los trabajos de aislamiento de cepas de heces humanas realizados por Park et al. (2002). Según la DO, los porcentajes de sobrevivencia fueron menores a los hallados por Tulumoglu et al. (2013), quienes sometieron a las cepas a un proceso de incubación de 2 horas, mientras que en el presente estudio fueron realizadas a 24 horas. Sin embargo, cuando se seleccionaron las 11 cepas más tolerantes al pH bajo durante 24 horas y sometieron a la misma prueba por dos horas, las cepas mostraron crecimiento de 7 log $\mathrm{UFC} / \mathrm{mL}$, valores similares encontrados por otros investigadores. Park et al (2002) igualmente al aislar cepas de heces de bebes obtuvieron un crecimiento de hasta 7 log UFC/ mL en medio a pH 3. 
Actividad antimicrobiana.- Jabbari et al. (2017), encontraron algunas cepas de Lactobacillus plantarum que no muestran actividad antimicrobiana contra $E$. coli y en algunos casos el halo fue más pequeño que otros patógenos. Por su parte Saxelin (1997), señala que determinadas cepas de lactobacilos tienen actividad antimicrobiana contra Salmonella y E. coli, sin embargo, en el presente estudio la cepa 7(1) inhibió a dos cepas patógenas de $E$. coli (ATCC25922 y ATCC35218)

Messensy (2002) señalan que varias bacterias ácido lácticas producen sustancias inhibitorias denominados bacteriocinas como bavaricina y plantaricina ST31, pero estas sustancias son diferentes de los ácidos orgánicos. Shin et al. (2008) hallaron genes de enterocina A y enterocina B en Enterococos faecium SH 632 que mostraron actividad inhibitoria contra Listeria monocitogenes, siendo además considerado como candidato potencial para ser usado como probióticos.

Identificación de las cepas aisladas.- La cepa 5(2) especie identificada con similaridad de $100 \%$ a Lactobacillus plantarum subsp. plantarum ATCC 14917 refuerza lo encontrado por Rodríguez (2009) quien aisló Lactobacillus plantarum de heces de niños. Este lactobacilo es considerado como probiótico GRAS (GenBank YJ Jin2018) y actualmente se está utilizando en alimentos para humanos y animales (Rodríguez 2009). Jabbari et al. (2017) aislaron Lactobacillus plantarum con características probióticas de queso tradicional, esta bacteria es la más utilizada para la producción de alimentos fermentados. Por lo tanto, esta bacteria al tener $100 \%$ de similaridad con Lactobacillus plantarum subsp. plantarum ATCC $14917^{\mathrm{T}}$ y ser considerada como cepa GRAS se sugiere su uso como bacteria probiótica en productos fermentados, mientras que la cepa 15 necesita ser sometidas a más pruebas para el uso como cultivo para la producción de productos fermentados de consumo humano.

La cepa 15 especie identificada con similaridad de 99.93\% a Enterococcus faecium CGMCC $1.2136^{\mathrm{T}}$ es aislado frecuentemente de las heces de humano y animales y son mucho más prevalentes en humanos que en el ganado, como los cerdos y las ovejas (Franz et al. 1999). Los enterococos tienen muchos rasgos positivos apreciados en la fermentación y conservación de los alimentos, y también pueden servir como probióticos (Ingolf 2014). Actualmente, el Enterococcus faecium CGMCC 1.2136 se utiliza en la fabricación de yogur en china (Ren et al. 2016). Foulquie et al. (2006) señalan que los enterococos se han utilizado como probióticos debido a sus posibles capacidades de promover buena salud. Así mismo los enterococos productores de bacteriocina son aislados de alimentos, heces y del tracto gastrointestinal de humanos y animales, pero también pueden aislarse de otras fuentes como las heces de bebés sanos y estos parecen ser ricos en enterococos que producen bacteriocina, por lo que es probable que la mayoría de los enterococos se originen en el tracto digestivo de humanos y animales (Ingolf 2014). Vélez (2014) por su parte aisló, lactococos y lactobacilos con características probióticas de leche porcina, por lo que también se pueden encontrar en le- che. Sin embargo, es necesario realizar pruebas adicionales para determinar el uso de la cepa 15 en productos fermentados para humanos.

Cinética de las cepas en leche.- En este trabajo, se necesitó tres horas de fermentación para que la cepa 5(2) pase de $5 \log \mathrm{UFC} / \mathrm{mL}$ a $7 \log \mathrm{UFC} / \mathrm{mL}$. Esto difie de lo reportado por Rodríguez (2009), quien demoró 10 horas para incrementar un ciclo de 6 a $7 \log \mathrm{UFC} / \mathrm{mL}$ al trabajar con cepas de Lactobacillus plantarum aislados de heces de niños y sembrados en leche. Sin embargo, transcurrido 24 horas de incubación, la cepa 5(2) alcanzó un ciclo menos de los hallados en 24 horas por Rodríguez (2009), debido probablemente a que la carga bacteriana inicial fue de 6 y no de $5 \mathrm{Log} \mathrm{UFC} / \mathrm{mL}$. La cepa 15, igualmente en 3 horas pasó de 5 a $7 \log \mathrm{UF} / \mathrm{mL}$ y a las 24 horas alcanzó el desarrollo de $8 \log \mathrm{UFC} / \mathrm{mL}$, mostrando buena cinética de crecimiento.

Variación de pH en la fermentación de leche.- La cepa 5(2) fermentó la leche alcanzando valores de pH 5.4 a las 3 horas de fermentación debido a la multiplicación de las bacterias por el consumo de la lactosa de la leche como fuente de energía. El pH 4.1 de la leche a las 12 horas de fermentación es fundamental para evitar el crecimiento de microorganismos patógenos. Aguilar y Cold (2011) demostraron que la viabilidad de E. coli disminuyó en un $100 \%$ cuando los valores de pH alcanzaron 4.0 por fermentación con L. plantarum LB279 en caldo MRS. Por lo tanto, al inocular con flora microbiológica que sea competitiva, inocua y no patógena, como las BAL, puede reducir el pH e inhibir la proliferación de patógenos y mejor si está alrededor de pH 5 (FAO/OMS 1997).

La cepa 15 después de 3 horas de fermentación alcanzó un pH de 5.3 partiendo con un valor más alto que la cepa 5(2), esto sugiere que posee buena actividad para producción de ácido láctico y ácidos orgánicos (Rahman 2013). Estos resultados indican que la cepa 15 tiene buena actividad y mayor velocidad de arranque de fermentación, característica importante cuando se quiere tener producción de ácido láctico en menor tiempo.

Sarantinopoulos et al. (2003) observaron el co-metabolismo de glucosa y citrato al cultivar en caldo MRS una cepa de E. faecium, tanto en condiciones aeróbicas como anaeróbicas. La fermentación del citrato por esta cepa es importante por la producción de diacetilo y acetaldehído, los cuales dan muy buenas características organolépticas a los productos lácteos fermentados. Aymerich et al. (2000) indican que las cepas de Enterococcus son competitivas durante la fermentación de la carne e inhibe fuertemente el crecimiento de Listeria spp. y al no producir sabores desagradables, el enterococo puede ser adecuado para la adición a la carne en calidad de cultivos para mejorar la seguridad alimentaria. Por otra parte, el ácido láctico producido por la cepa 15 , actualmente ha tomado gran importancia a nivel mundial por su utilidad. Rahman et al. (2013), mencionan que el ácido poliláctico biodegradable que sustituye a los plásticos derivados del petróleo, se obtiene por la producción de ácido láctico mediante el uso de Enterococcus faecium. 
De acuerdo a los resultados obtenidos en este estudio se puede concluir que las heces de neonatos humanos nacidos en el INMP de Lima posee bacterias acido lácticas con potencial probiótico como tolerancia a ácido, resistencia a la bilis, actividad antimicrobiana y fermentan la leche.

\section{Literatura citada}

Aguilar C. \& B. Cold. 2008. Inhibición del crecimiento de Escherichia coli por bacterias ácido lácticas: presencia de quórum sensing?. Revista de la Asociación Colombiana de Ciencia y Tecnología de Alimentos. 13(13):1-9.

Ávila J., M. Ávila, B. Tovar, et al. 2010. Capacidad probiótica de cepas del genero Lactobacillus extraídas del tracto intestinal de animales de granja. Revista Científica FCVLUZ. XX(2):161-9.

Aymerich T., M.G. Artigas, M. Garriga et al. 2000. Effect of sausage ingredients and additives on the production of enterocin A and B by Enterococcus faecium CTC492. Optimization of in vitro production and anti-listerial effect in dry fermented sausages. Journal Applied Microbiology. 88(4):686-94.

Boke H., B. Aslim y G. Alp. 2010. The role of resistance to bile salts and acid tolerance of exopolysaccharides (EPSS) produced by yogurt starter bacteria. Archives of Biological Sciences. 62(2):323-8. Doi: 10.2298/ ABS1002323B

Brink M., S. Todorov, J.H. Martin et al. 2006. The effect of prebiotics on production of antimicrobial compounds, resistance to growth at low $\mathrm{pH}$ and in the presence of bile, and adhesion of probiotic cells to intestinal mucus. Journal of Applied Microbiology. 100(4):813-20. doi: 10.1111/j.1365-2672.2006.02859.x

Clark P. \& J. Martin. 1994. Selection of Bifidobacteria for use as dietary adjuncts in cultured dairy foods. II. Tolerance to simulated $\mathrm{pH}$ of human stomachs. Journal of Cultured Dairy Products. 29:18-21.

Cueto C. \& S. Aragón. 2012. Evaluación del potencial probiótico de bacterias ácido lácticas para reducir el colesterol in vitro. Scientia Agropecuaria. 1(2012):45-50.

Erkkila S. \& E. Petaja. 2000. Screening of commercial meat starter cultures at low $\mathrm{pH}$ and in the presence of bile salts for potential probiotic use. Meat Science. 55(3):297300 .

FAO/OMS (Organización de las Naciones Unidas para la Agricultura y Alimentación / Organización Mundial de la Salud). 1997. Programa conjunto FAO/OMS sobre normas alimentarias. <http://www.fao.org/docrep/ meeting/005/AC338F/AC338F00.htm\#TOC>. (Acceso $12 / 12 / 2015)$.

Fisher K. \& C. Phillips. 2009. The ecology, epidemiology and virulence of Enterococcus. Microbiology. $155(\mathrm{Pt}$ 6):1749-57. doi: 10.1099/mic.0.026385-0.

Franz C.M., M.E. Stiles, K.H. Schleifer, et al. 2003. Enterococci in foods-a conundrum for food safety. International Journal Food Microbiology. 88(2-3):105-22.

Guarne F. 2007. Papel de la flora intestinal en la salud y en la enfermedad. Nutrición Hospitalaria. 22(2):14-9.

Ibañez E.S. 2017. Microbiota en leche Humana. 38.Congreso Argentino de pediatría.Cordova.
Ingolf F., B. Dzung, Y. Ike. 2014. Enterococcal Bacteriocins and Antimicrobial Proteins that Contribute to Niche Control. Enterococci: From commensals to leading causes of drug resistant infection. Boston: Massachusetts Eye and Ear Infirmary. <https://www.ncbi.nlm.nih.gov/ books/NBK190428/>. (Acceso 15/06/2018).

Jabbari V., M.S. Khiabani, R.R. Mokarram, et al. 2017. Lactobacillus plantarum as a Probiotic Potential from Kouzeh Cheese (Traditional Iranian Cheese) and Its Antimicrobial Activity. Probiotics and Antimicrobial Proteins. 9(2):189-93. doi: 10.1007/s12602-017-9255-0.

Klaenhammer T.R. 1982. Microbiological considerations in selection and preparation of Lactobacillus strains for use as dietary adjuncts. Journal of Dairy Science. 65(7):1339-49. doi: 10.3168/jds.S00220302(82)82351-5.

Lara C. \& A. Burgos. 2012. Potencial probiótico de cepas nativas para uso como aditivos en la alimentación avícola. Revista Colombia de Biotecnología. 14(1):31-40.

La Rosa D., E. J. Gómez, N. Sánchez. 2014. La microbiota intestinal en el desarrollo del sistema inmune del recién nacido. Rev Cubana Pediatr 86(4)

Lee H.M. \& Y. Lee. 2008. A differential medium for lactic acid-producing bacteria in a mixed culture. Letters in Applied Microbiology. 46(6):676-81. doi: 10.1111/j.1472-765X.2008.02371. x

Leroy F., M.R. Foulquié y L. De Vuyst. 2003. Enterococcus faecium RZS C5, an interesting bacteriocin producer to be used as a co-culture in food fermentation. International Journal of Food Microbiology. 88(2-3):235-40.

Messens W. \& V.L. De. 2002. Inhibitory substances produced by Lactobacilli isolated from sourdoughs-a review. International Journal of Food Microbiology. 72(1-2):31-43.

Miranda M.G. 2008. Probióticos y micro nutrimentos ¿Son útiles para el tratamiento de la diarrea aguda?. Boletín Médico del Hospital Infantil de México. 65(3) México mayo-junio.

Moreno R., E. Salas, C. Pérez et al. 2011. Evaluación del potencial probiótico de lactobacilos aislados de heces de lactantes y leche materna. Medula, Revista de Facultad de Medicina, Universidad de Los Andes. 20(2):135-9.

OlivareS M, J. Moisés Laparra, Yolanda Sanz. 2013. Host genotype, intestinal microbiota and inflammatory disorders. doi.org/10.1017/S0007114512005521

Park Y.S., J.Y. Lee, Y.S. Kim, et al. 2002. Isolation and characterization of lactic acid bacteria from feces of newborn baby and from dongchimi. Journal of Agricology Food Chemistry. 50(9):2531-6.

Rahman M.A., Y. Tashiro y K. Sonomoto. 2013. Recent advances in lactic acid production by microbial fermentation processes. Biotechnology Advances. 31(6):877-902. doi: 10.1016/j.biotechadv.2013.04.002.

Ren X., M. Li y D. Guo. 2016. Enterococcus Xinjiangensis sp. nov., Isolated from Yogurt of Xinjiang, China. Current Microbiology. 73(3):374-8. doi: 10.1007/s00284016-1065-1

Rodríguez M. 2009. Aislamiento y selección de cepas del género Lactobacillus con capacidad probiótica e inmunomodeladora. Tesis, Doctorado en Genética y Microbiología. Facultad de Biociencias. Universidad Autónoma de Barcelona. [TDX, tesis]. <http://www. tdx.cat/bitstream/handle/10803/3931/mrg1de1. pdf?sequence $=1>$. Acceso 12/12/2017 
Sarantinopoulos P., L. Makras, F. Vaningelgem, et al. 2003. Growth and energy generation by Enterococcus faecium FAIR-E 198 during citrate metabolism. International Journal of Food Microbiology. 84(2):197-206. doi: 10.1016/S0168-1605(02)00421-X

Sánchez L, M. Omura, A. Lucas, et al. 2015. Cepas de Lactobacillus spp. con capacidades probióticas aisladas del tracto intestinal de terneros neonatos. Revista de Salud Animal. 37(2):94-104

Saxelin M. 1997. Lactobacillus GG-a human probiotic strain with thorough clinical documentation. Journal Food Reviews International. 13(2):293-313. doi: $10.1080 / 87559129709541107$

Suárez J.E. 2013. Microbiota autóctona, probióticos y prebióticos. Nutr. Hosp. vol.28 supl.1 Madrid ene. 2013

Tai N., J. Peng, F. Liu, et al. 2016. Microbial antigen mimics activate diabetogenic CD8 T cells in NOD mice. Journal of Experimental Medicine. 213(10):2129-46. doi:10.1084/jem.20160526

Vallejo M., Olivera N, Sequeiros C. y Marguet E. 2009. Actividad antilisteria de bactérias ácido lácticas aisladas de peces marinos. Analecta Veterinaria. 29(2):19-23.

Vélez J.M. 2014. Evaluación de actividad antimicrobiana de bactérias probióticas extraídas del calostro de cerdas de granjas del Aburrá sur. Tesis, Magíster en Biotecnología. Facultad de Ciencias, Universidad Nacional de Colombia [BDIGITAL, tesis] < http://bdigital.unal. edu.co/45835/1/32255433.2014.pdf>. (Acceso 04/08/2017).

Versalovic J., T. Koeuth y J. Lupski. 1991. Distribution of repetitive DNA sequences in eubacteria and application to fingerprinting of bacterial genome. Nucleic Acids Research. 19(24):6823-31.
Vizoso Pinto MG, Franz CM, Schillinger U, Holzapfel WH.2006. lactobacillus spp. With in vitro probiotic propierties from human faeces and traditional fermented products. DOI: 10.1016/j.ijfoodmicro.2006.01.029

Yadav R., A.K. Puniya y P. Shukla. 2016. Probiotic properties of Lactobacillus plantarum RYPR1 from an indigenous fermented beverage raabadi. Frontiers in Microbiology. 7:1683. doi: 10.3389/fmicb.2016.01683

\section{Agradecimientos}

Al Laboratorio de Ecología Microbiana y Biotecnología, Dpto. de Biología de la Facultad de Ciencias, Laboratorio de la Facultad de Zootecnia de la Universidad Nacional Agraria La Molina, y a la Universidad Nacional del Centro de Perú.

Información sobre los autores:

EP realizo la concepción y diseño del artículo, la recolección de muestras aislamiento de cepas, instalación de ensayos y redacción de artículo. RS realizo extracción de ADN, detección de calidad de la misma y redacción de artículo. AS realizo procesamiento de datos y actividad antimicrobiana. DZ realizo el monitoreo de los ensayos de la parte molecular e interpretación y CV realizo monitoreo de la fermentación de la leche y análisis e interpretación de los datos.

\section{Conflicto de intereses:}

Los autores no incurren en conflictos de intereses.

Aspectos éticos / legales:

Proyecto aprobado por el Comité de Evaluación Metodológica y Estadística en la investigación y el Comité de Ética en Investigación del Instituto Nacional Materna Perinatal. Expediente Nº17411-16.

Fuentes de financiamiento:

Los autores declaran no haber tenido una entidad financiadora específica. 
